\title{
A $\mathrm{S}=1 / 2$ vanadium-based geometrically frustrated spinel system $\mathrm{Li}_{2} \mathrm{ZnV}_{3} \mathrm{O}_{8}$
}

\author{
T. Chakrabarty ${ }^{*}$ \\ Department of Physics, IIT Bombay, Powai, Mumbai 400076, India \\ A. V. Mahajan ${ }^{\dagger}$ \\ Department of Physics, IIT Bombay, Powai, Mumbai 4000\%6, India \\ B. Koteswararao \\ (CeNSCMR), Department of Physics and Astronomy, and Institute of Applied Physics, \\ Seoul National University, Seoul 151-747, South Korea
}

\begin{abstract}
We report the synthesis and characterization of $\mathrm{Li}_{2} \mathrm{ZnV}_{3} \mathrm{O}_{8}$, which is a new $\mathrm{Zn}$-doped $\mathrm{LiV}_{2} \mathrm{O}_{4}$ system containing only tetravalent vanadium. A Curie-Weiss susceptibility with a Curie-Weiss temperature of $\theta_{C W} \approx-214 \mathrm{~K}$ suggests the presence of strong antiferromagnetic correlations in this system. We have observed a splitting between the zero-field cooled ZFC and field cooled FC susceptibility curves below $6 \mathrm{~K}$. A peak is present in the ZFC curve around $3.5 \mathrm{~K}$ suggestive of spin-freezing . Similarly, a broad hump is also seen in the inferred magnetic heat capacity around $9 \mathrm{~K}$. The consequent entropy change is only about $8 \%$ of the value expected for an ordered $S=1 / 2$ system. This reduction indicates continued presence of large disorder in the system in spite of the large $\theta_{C W}$, which might result from strong geometric frustration in the system. We did not find any temperature $T$ dependence in our ${ }^{7} \mathrm{Li}$ nuclear magnetic resonance NMR shift down to $6 \mathrm{~K}$ (an abrupt change in the shift takes place below $6 \mathrm{~K}$ ) though considerable $T$-dependence has been found in literature for $\mathrm{LiV}_{2} \mathrm{O}_{4}-$ undoped or with other $\mathrm{Zn} / \mathrm{Ti}$ contents. Consistent with the above observation, the ${ }^{7} \mathrm{Li}$ nuclear spin-lattice relaxation rate $1 / T_{1}$ is relatively small and nearly $T$-independent except a small increase close to the freezing temperature, once again, small compared to undoped or $10 \% \mathrm{Zn}$ or $20 \%$ Ti-doped $\mathrm{LiV}_{2} \mathrm{O}_{4}$.
\end{abstract}

PACS numbers: 75.10.Pq,75.40.Cx,76.60.-k

\section{INTRODUCTION}

Cubic spinel materials $\mathrm{AB}_{2} \mathrm{O}_{4}$ with non-magnetic ions at the $\mathrm{A}$-site and magnetic ions at the $\mathrm{B}$-site are interesting due to the existence of a geometrically frustrated ${ }^{1 / 2}$ B-sublattice (corner-shared tetrahedra) in them. Perhaps the most studied compound in this category is $\mathrm{LiV}_{2} \mathrm{O}_{4}$ which exhibits the $d$-electron derived heavy fermi liquid behaviour ${ }^{3}$-5 . In the motivation of Villain's work ${ }^{6}$ $\mathrm{LiV}_{2} \mathrm{O}_{4}$ doped with a non-magnetic impurity at the $\mathrm{V}$ site or with a magnetic impurity at the A-site have also been studied extensively 7 11. Likewise, the structure of $\mathrm{Na}_{4} \mathrm{Ir}_{3} \mathrm{O}_{8}$ or $\left[\mathrm{Na}_{1.5}\left(\mathrm{Na}_{0.25} \mathrm{Ir}_{0.75}\right)_{2} \mathrm{O}_{4}\right]$ is also derived from the spinel structure 12 . Here, the Na1.5 are at the A-sites whereas $\mathrm{Ir}$ and $\mathrm{Na}$ at the B-sites have distinct positions and the Ir ions form corner shared triangles in three dimensions. This has been dubbed as the hyperkagome lattice and is proposed to have a quantum spin liquid ground state.

Motivated by the above we have been searching for new $S=1 / 2$ spinel systems which might exhibit interesting properties. There exists $\mathrm{Li}_{2} \mathrm{ZnTi}_{3} \mathrm{O}_{8}$ which has the same space group as $\mathrm{Na}_{4} \mathrm{Ir}_{3} \mathrm{O}_{8}$, i.e., $P_{43} 32$ and the $\mathrm{Zn}$ and the $\mathrm{Ti}$ ions are at distinct B-sites therefore the $\mathrm{Ti}$ ions form a hyperkagome lattice. This compound is unfortunately nonmagnetic due to the +4 oxidation state of the titanium. We therefore set out to prepare $\mathrm{Li}_{2} \mathrm{ZnV}_{3} \mathrm{O}_{8}$ which would have a $S=1 / 2\left(\mathrm{~V}^{4+}\right)$ hyperkagome lattice, in the case in which it formed with the same structure as $\mathrm{Li}_{2} \mathrm{ZnTi}_{3} \mathrm{O}_{8}$. In this paper we report the properties of a new $\mathrm{V}$-based spinel $\mathrm{Li}_{2} \mathrm{ZnV}_{3} \mathrm{O}_{8}$. Our results show that $\mathrm{Li}_{2} \mathrm{ZnV}_{3} \mathrm{O}_{8}$ does not form in the $P_{43} 32$ space group and hence is not isostructural to $\mathrm{Li}_{2} \mathrm{ZnTi}_{3} \mathrm{O}_{8}$. In fact, $\mathrm{Li}_{2} \mathrm{ZnV}_{3} \mathrm{O}_{8}$ forms in the $F d-3 m$-s space group where there is no unique $\mathrm{B}$-site and it is shared by two ions. So, $\mathrm{Li}_{2} \mathrm{ZnV}_{3} \mathrm{O}_{8}$ could be written as $\left[\mathrm{Li}\left(\mathrm{Zn}_{0.25} \mathrm{~V}_{0.75}\right)_{2} \mathrm{O}_{4}\right]_{2}$ or $\left[\left(\mathrm{Li}_{0.5} \mathrm{Zn}_{0.5}\left(\mathrm{Li}_{0.25} \mathrm{~V}_{0.75}\right)_{2} \mathrm{O}_{4}\right]_{2}\right.$ or possibly with a site occupation which is in between the two. In the former site assignment, $\mathrm{Li}$ is at the tetrahedral A-site and the $\mathrm{Zn}$ and $\mathrm{V}$ ions share the octahedral B-sites while in the latter, $\mathrm{Li}$ and $\mathrm{Zn}$ are at the tetrahedral A-sites and $\mathrm{Li}$ and $\mathrm{V}$ ions share the octahedral B-sites. Consequently, although the B-sublattice forms a geometrically frustrated network, in both the cases only $75 \%$ of its sites are (statistically) occupied by the magnetic $\mathrm{V}^{4+}$ ions. Due to this site-sharing at the B-site, the frustration effect is likely diluted and might result in a spin-disordered (frozen) state at low temperature. In $\mathrm{LiV}_{2} \mathrm{O}_{4}$ the $\mathrm{V}^{4+} / \mathrm{V}^{3+}$ ratio is 1 and the system shows heavy fermionic behavior. When a nonmagnetic impurity (Zn,Ti) is doped at the B-site or at the A-site of the spinel then the system exhibits spin freezing or spin glass behaviour at a temperature which depends on the doping element and its concentration 79|13. In all these above mentioned doped $\mathrm{LiV}_{2} \mathrm{O}_{4}$ systems there is likely a mixture of $\mathrm{V}^{4+}$ and $\mathrm{V}^{3+}$ ions. In contrast, in $\mathrm{Li}_{2} \mathrm{ZnV}_{3} \mathrm{O}_{8}$ the effective valence of vanadium is 4 , which to our knowledge is the first doped- $\mathrm{LiV}_{2} \mathrm{O}_{4}$ system but still with only $\mathrm{V}^{4+}(S=1 / 2)$ ions.

The magnetic susceptibility of $\mathrm{Li}_{2} \mathrm{ZnV}_{3} \mathrm{O}_{8}$ is found to be of the Curie-Weiss form above $150 \mathrm{~K}$ with an effective moment close to that expected for $S=1 / 2$. The 
Curie-Weiss temperature $\theta_{C W}=-214 \mathrm{~K}$ is indicative of strong antiferromagnetic $(\mathrm{AF})$ interactions. This value of the Curie-Weiss temperature is quite high compared to the earlier doped $\mathrm{LiV}_{2} \mathrm{O}_{4}$ systems indicating much stronger correlation between the magnetic $\mathrm{B}$-sites of the system. For the series $\mathrm{Li}_{1-x} \mathrm{Zn}_{x} \mathrm{~V}_{2} \mathrm{O}_{4}$ the maximum value of $\theta_{C W}$ is about $-68 \mathrm{~K}$ (for $\left.x=0.3\right)^{10}$ and for the series $\mathrm{Li}\left(\mathrm{V}_{1-y} \mathrm{Ti}_{y}\right)_{2} \mathrm{O}_{4}$ the maximum value of $\theta_{C W}$ is about $-56 \mathrm{~K}$ (for $y=0.3) 10$. A difference between the zero-field cooled (ZFC) and field-cooled (FC) susceptibilities is observed by us for $\mathrm{Li}_{2} \mathrm{ZnV}_{3} \mathrm{O}_{8}$, below about $6 \mathrm{~K}$, suggesting random magnetic interactions and spinfreezing. A magnetic contribution to the heat capacity is present at low temperatures though with a small entropy change, suggesting a highly degenerate ground state. We also report ${ }^{7} \mathrm{Li}$ NMR spectra and spin-lattice relaxation rate $\left(1 / T_{1}\right)$ measurements and compare these with the results for pure and other $\mathrm{Zn} / \mathrm{Ti}$ doped $\mathrm{LiV}_{2} \mathrm{O}_{4}$.

\section{EXPERIMENTAL DETAILS}

$\mathrm{Li}_{2} \mathrm{ZnV}_{3} \mathrm{O}_{8}$ was prepared by standard solid state reaction methods. First we prepared $\mathrm{V}_{2} \mathrm{O}_{3}$ by reducing $\mathrm{V}_{2} \mathrm{O}_{5}$ (Aldrich-99.99\%) in hydrogen atmosphere at $650^{\circ} \mathrm{C}$ for 16 hours. Then we prepared $\mathrm{VO}_{2}$ by mixing this $\mathrm{V}_{2} \mathrm{O}_{3}$ with $\mathrm{V}_{2} \mathrm{O}_{5}$ in a 1:1 molar ratio, pelletizing and firing in dynamical vacuum (better than $10^{-5}$ mbar) at $800^{\circ} \mathrm{C}$ for 24 hours. In the next step we mixed $\mathrm{Li}_{2} \mathrm{CO}_{3}$ (Alpha Aesar 99.995\% purity), ZnO (Aldrich $99.99 \%$ purity) and $\mathrm{VO}_{2}$, pelletized it, and then fired in a tubular furnace at $650^{\circ} \mathrm{C}$ for 28 hours under a dynamical vacuum (better than $10^{-5}$ mbar). X-ray diffraction (xrd) patterns were collected with a PANalytical x-ray diffractometer using $\mathrm{Cu} \mathrm{K} \alpha$ radiation $(\lambda=1.54182)$. The Rietveld refinement of the xrd pattern (see Fig 1) was carried out using the "Fullprof" 14 software. The lattice parameter was found to be $8.332 \AA$ in the $F d$ $3 \mathrm{~m}$ space group. The formula of $\mathrm{Li}_{2} \mathrm{ZnV}_{3} \mathrm{O}_{8}$ could be written in two different ways, $\left[\mathrm{Li}\left(\mathrm{Zn}_{0.25} \mathrm{~V}_{0.75}\right)_{2} \mathrm{O}_{4}\right]_{2}$ and $\left[\left(\mathrm{Li}_{0.5} \mathrm{Zn}_{0.5}\left(\mathrm{Li}_{0.25} \mathrm{~V}_{0.75}\right)_{2} \mathrm{O}_{4}\right]_{2}\right.$. Out of the two possible site arrangements in $\mathrm{Li}_{2} \mathrm{ZnV}_{3} \mathrm{O}_{8}$, the second one is possibly the right site assignment since $\mathrm{Zn}$ is known to prefer the tetrahedral site in spinels!15. In both these possibilities $\mathrm{V}$ is at the $\mathrm{B}$-site. We refined the xrd data of $\mathrm{Li}_{2} \mathrm{ZnV}_{3} \mathrm{O}_{8}$ to determine the site occupation. The best refinement was obtained with the formula $\left[\left(\mathrm{Li}_{0.46} \mathrm{Zn}_{0.43}\right)\left(\mathrm{Li}_{0.24} \mathrm{~V}_{0.75}\right)_{2} \mathrm{O}_{4}\right]_{2}$ which imples some $\mathrm{Zn}$ deficiency at the A-site. The results of the refinements are shown in Table $\mathrm{I}$ below. The goodness of the Rietveld refinement is defined by the following parameters. $\mathrm{R}_{p}=19.6 \%, \mathrm{R}_{w p}=16.9 \%, \mathrm{R}_{e x p}=5.30 \%$, and $\chi^{2}=11.3$. There were some extra phases of $\mathrm{LiV}_{2} \mathrm{O}_{5}, \mathrm{Li}_{3} \mathrm{VO}_{4}$ and $\mathrm{VO}_{2}$ present at the $1 \%$ level. The Rietveld refinement is done considering all the 4 phases.

From ICP-AES (inductively coupled plasma-atomic emission spectroscopy) we determined the ratio of lithium with zinc and vanadium. From this analysis we
Table I: Atomic positions in $\mathrm{Li}_{2} \mathrm{ZnV}_{3} \mathrm{O}_{8}$

\begin{tabular}{|c|c|c|c|c|}
\hline \multirow{2}{*}{ Atoms } & \multicolumn{3}{|c|}{ Co-ordinates } & Occupancy \\
\cline { 2 - 5 } & $\mathrm{x} / \mathrm{a}$ & $\mathrm{y} / \mathrm{b}$ & $\mathrm{z} / \mathrm{c}$ & \\
\hline Li1(8a) & 0.000 & 0.000 & 0.000 & 0.46 \\
\hline Zn1(8a) & 0.000 & 0.000 & 0.000 & 0.43 \\
\hline Li2(16d) & 0.625 & 0.625 & 0.625 & 0.24 \\
\hline V(16d) & 0.625 & 0.625 & 0.625 & 0.75 \\
\hline O1(32e) & 0.594 & 0.594 & 0.594 & 1.00 \\
\hline
\end{tabular}

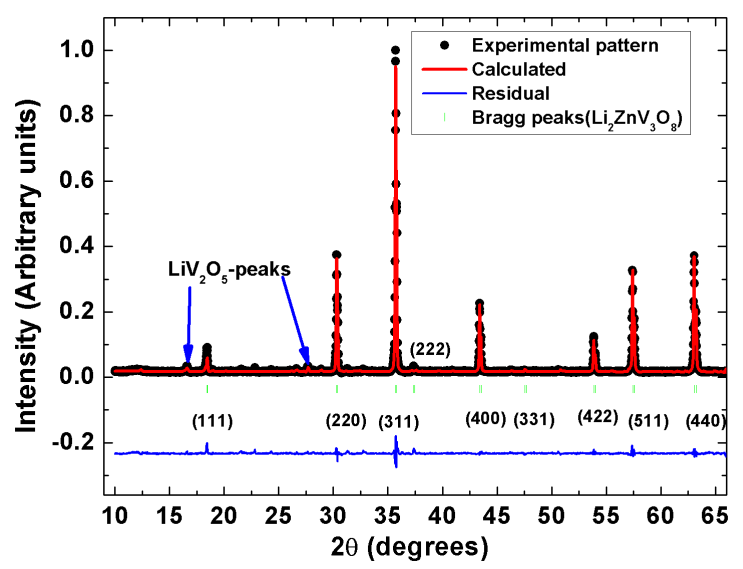

Figure 1: Powder diffraction pattern of $\mathrm{Li}_{2} \mathrm{ZnV}_{3} \mathrm{O}_{8}$ is shown along with its Bragg peak positions; The black points are the experimental data, the red solid line is the "Fullprof" generated refinement pattern, the green markers are the Bragg peak positions for $F d-3 m-s$ space group and the blue points represent the "experimental - calculated" intensity pattern. $\mathrm{LiV}_{2} \mathrm{O}_{5}$ impurity peaks are shown by blue arrows.

found the molar formula unit of the compound to be $\mathrm{Li}_{1.98} \mathrm{Zn}_{0.86} \mathrm{~V}_{3} \mathrm{O}_{8}$. This result is consistent with our "Fullprof" refinement since there also we found that zinc is deficient at the A-site.

In the structure of $\mathrm{Li}_{2} \mathrm{ZnV}_{3} \mathrm{O}_{8}$ the B-sites (shared by $\mathrm{Zn}^{2+}$ and $\mathrm{V}^{4+}$ in the 1:3 ratio) form a corner-shared tetrahedral network [see Fig,2. In case of random/statistical occupation of the B sites by $\mathrm{Zn}$ and $\mathrm{V}$, there are going to be missing magnetic atoms in the triangular network. This disruption/dilution of the corner-shared tetrahedral network is likely to lead to relieving of frustration and this might then lead to a spin-glass like state at low temperature.

The temperature dependence of magnetization $M$ was measured in a $1 \mathrm{kOe}$ magnetic field $H$ in the temperature range 2-300 $\mathrm{K}$ using a vibrating sample magnetometer (VSM) attached with a Quantum Design Physical Property Measurement System (PPMS). The temperature dependence of heat capacity has also been measured in the temperature range of 2-270 $\mathrm{K}$ using the heat capacity attachment of a Quantum Design PPMS. The ${ }^{7} \mathrm{Li}$ (gyromagnetic ratio $\gamma / 2 \pi=16.54607 \mathrm{MHz} / \mathrm{kOe}$ and nuclear spin $I=3 / 2$ ) NMR measurements using a fixed field 


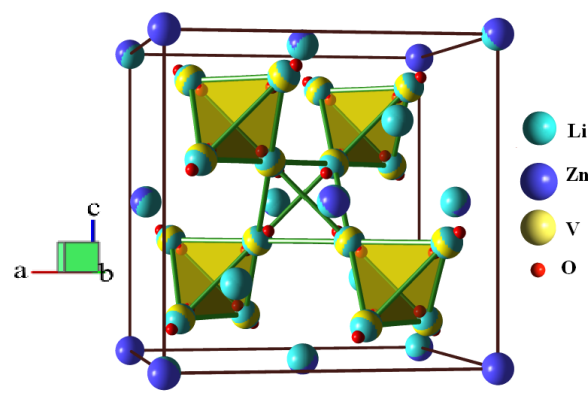

Figure 2: Unit cell of $\mathrm{Li}_{2} \mathrm{ZnV}_{3} \mathrm{O}_{8}$. The $\mathrm{Li}^{+}$, $\mathrm{Zn}^{2+}, \mathrm{V}^{4+}, \mathrm{O}^{2-}$ ions are shown in indigo, blue, yellow and red color respectively. The B-sites form a corner-shared tetrahedral network but are shared by $\mathrm{Li}^{+} / \mathrm{Zn}^{2+}$ and $\mathrm{V}^{4+}$.

of $93.9543 \mathrm{kOe}$ have been carried out. The ${ }^{7} \mathrm{Li}$ nuclear spin-lattice relaxation was measured by the saturation recovery method using a $n \times \pi / 2-t-\pi / 2-\tau-\pi$ pulse sequence with $\tau=90 \mu s$ and variable $t$. A comb sequence with $n$ (between 5 and 10) pulses was used to obtain good saturation.

\section{RESULTS AND DISCUSSIONS}

The reciprocal of the susceptibility is plotted with temperature in Fig. 3. We fit the data to $1 /\left(\chi-\chi_{0}\right)=$ $\left(T-\theta_{C W}\right) / C$ in the range $190-300 \mathrm{~K}$, where we took $\chi_{0}$ as the sum of the core diamagnetic susceptibilites for all the atoms in $\mathrm{Li}_{2} \mathrm{ZnV}_{3} \mathrm{O}_{8}{ }^{16}$ and the Van-Vleck paramagnetic susceptibility $\chi_{V V}=2.048 \times 10^{-4} \mathrm{~cm}^{3} /$ mole based on the vanadium-based $S=1 / 2$ spinel compound $\mathrm{LiV}_{2} \mathrm{O}_{4}{ }^{17}$ From the Curie constant obtained from the fit $\left(0.30 \mathrm{~cm}^{3} \mathrm{~K} /\right.$ mole $\left.\mathrm{V}^{4+}\right)$, the effective number of Bohr magnetons is found to be 1.55 which is slightly smaller than the value for a $S=1 / 2$ system $\left(\mu_{\text {eff }}=1.73 \mu_{\mathrm{B}}\right)$. Whereas no sharp anomaly is seen in the susceptibility data down to $2 \mathrm{~K}$, a difference betwen the ZFC and FC susceptibilities is seen below about $6 \mathrm{~K}$ suggestive of freezing of moments or glassy behaviour. Such ZFC/FC bifurcation has been seen in $\mathrm{Zn}$ or $\mathrm{Ti}$ doped $\mathrm{LiV}_{2} \mathrm{O}_{4}$ around the same temperature. The asymptotic CurieWeiss temperature is large and negative $\left(\theta_{C W} \simeq-214\right.$ $\mathrm{K}$ ), in comparison to the freezing temperature, indicating strong antiferromagnetic interactions. Note that the peak of the ZFC curve (at $T \sim 3.5 \mathrm{~K}$ ) is less than the temperature where the $\mathrm{ZFC} / \mathrm{FC}$ bifurcation starts. The $\mathrm{ZFC} / \mathrm{FC}$ split is possibly linked with the onset of spincluster formation and the peak of the ZFC curve indicates the freezing temperature $\left(T_{f}\right)$. These anomalies point to the fact that the freezing phenomena might set in already above $T_{f}$. Similar kind of behavior has also been observed in $\mathrm{Li}_{1-x} \mathrm{Zn}_{x} \mathrm{~V}_{2} \mathrm{O}_{4}$ (for $x \geq 0.8^{13}$ ) and in $\mathrm{Li}\left(\mathrm{V}_{1-y} \mathrm{Ti}_{y}\right)_{2} \mathrm{O}_{4}$ (for $y=0.2 \frac{10}{}$ ) The inherent geometric frustration of the spinel system coupled with the random occupation of the $\mathrm{B}$ sites by magnetic $\mathrm{V}^{4+}$ and nonmagnetic $\mathrm{Li}^{+}$is sug-

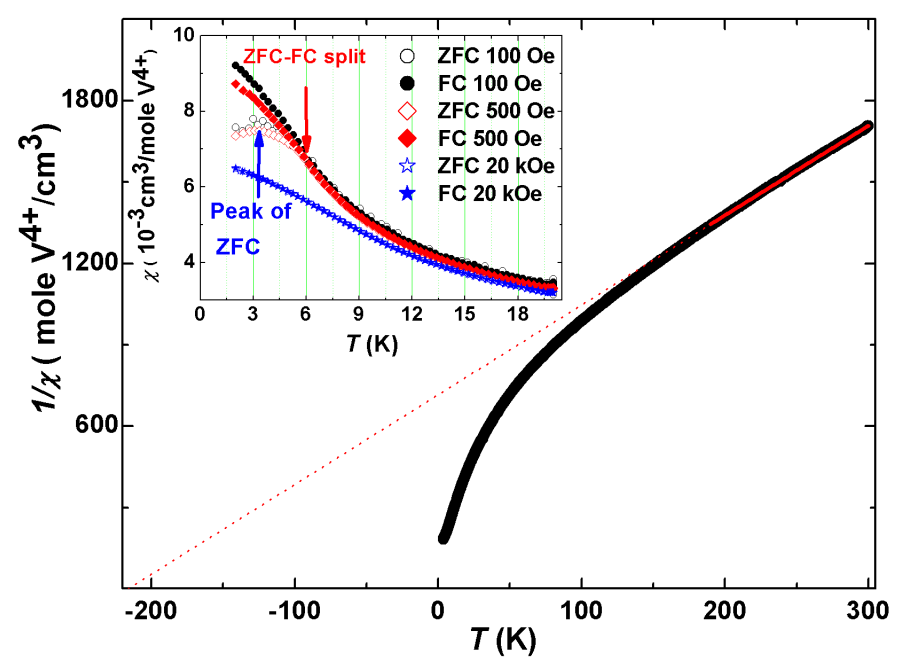

Figure 3: Temperature dependence of inverse susceptibility $1 /\left(\chi-\chi_{0}\right)$ is shown for $H=10 \mathrm{kOe}$. The red line shows the Curie-Weiss fit in the temperature range of 190-300 K and the dotted line is its extrapolation. The inset shows the bifurcation between the ZFC and FC curves below about 6 $\mathrm{K}$. The ZFC and FC data are shown for various fields. The peak of the ZFC curve and the starting point of $\mathrm{ZFC} / \mathrm{FC}$ bifurcation have been pointed out by the blue and red arrow, respectively.

gested to be responsible for the observed susceptibility behaviour. Note that in comparison, the $\theta_{C W}$ values for the $\mathrm{Li}\left(\mathrm{V}_{1-y} \mathrm{Ti}_{y}\right)_{2} \mathrm{O}_{4}(y=0.05-1)$ system are less than $100 \mathrm{~K}$.10. In our ac susceptibility measurements as well, a hump-like anomaly was observed at $4 \mathrm{~K}$ in the real part of the susceptibility $\chi^{\prime}$ (see Fig, 4). In the range of frequencies of the ac field considered by us $(20-1000 \mathrm{~Hz})$, no shift in the peak position of $\chi^{\prime}$ was observed. This is different from the conventional spin-glass behavior observed in the $\mathrm{Zn}$-doped (at A-site) of $\mathrm{Li}_{1-x} \mathrm{Zn}_{x} \mathrm{~V}_{2} \mathrm{O}_{4}{ }^{7}$. This suggests that there exists some kind of spin freezing in this compound but it is not the conventional spin glass behaviour.

The heat capacity data of $\mathrm{Li}_{2} \mathrm{ZnV}_{3} \mathrm{O}_{8}$ are shown in Fig.5. It is clear from the figure that no sign of longrange ordering is observed down to $2 \mathrm{~K}$. At around $5 \mathrm{~K}$ a small hump is seen in the $C_{\mathrm{p}} / T$ data and it changes slightly with the change in the magnetic field however this change with magnetic field is not due to the Schottky effect. This hump in the heat capacity data is close to the temperature where ZFC and FC curves are split in the $\chi(T)$ vs. $T$ data. Since we do not have a suitable non-magnetic analogue for this system we tried to extract the magnetic specific heat of $\mathrm{Li}_{2} \mathrm{ZnV}_{3} \mathrm{O}_{8}$ by subtracting the lattice contribution using a combination of Debye and Einstein heat capacities, $C_{\text {Debye }}$ and $C_{\text {Einstein }}$, respectively. In the $T$-range $27-125 \mathrm{~K}$, the measured heat capacity $C_{P}$ could be fit with a combination of one Debye and two Einstein functions of the type given below where the coefficient $C_{d}$ stands for the relative weight of 


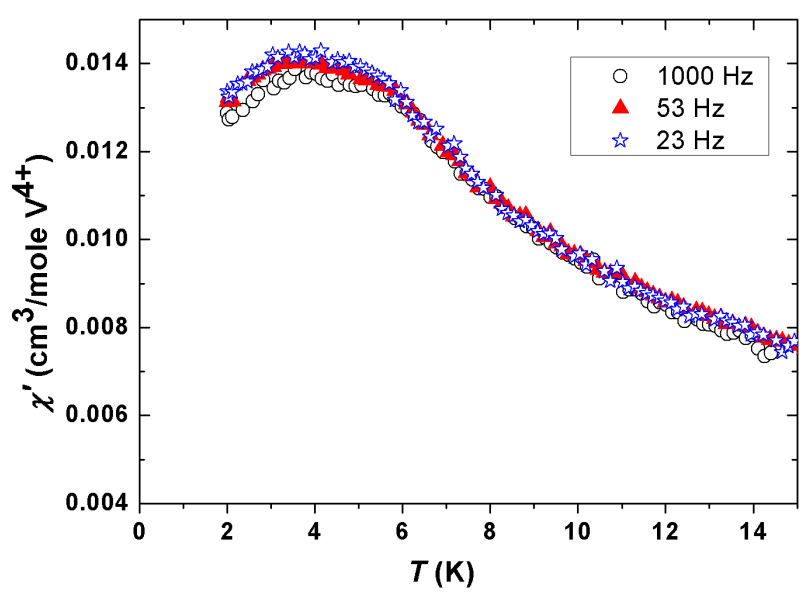

Figure 4: Temperature dependence of the $a c$ susceptibility $\left(\chi^{\prime}\right)$ measured in an $a c$ field of 1 Oe. The black, red and blue data points represent the measurements at $1000 \mathrm{~Hz}, 53 \mathrm{~Hz}$, and $23 \mathrm{~Hz}$ respectively. A hump-like anomaly is observed at $4 \mathrm{~K}$ in $\chi^{\prime}$ but no dependence on frequency has been observed.

the acoustic modes of vibration and the coefficients $C_{e_{1}}$ and $C_{e_{2}}$ are the relative weights of the optical modes of vibration.

$$
\begin{aligned}
& C_{\text {Debye }}=C_{d} \times 9 n R\left(T / \theta_{\mathrm{d}}\right)^{3} \int_{0}^{\theta_{\mathrm{d}} / T}\left(x^{4} e^{x} /\left(e^{x}-1\right)^{2}\right) d x \\
& C_{\text {Einstein }}=3 n R\left[\sum C_{e_{m}} \times \frac{x_{E_{m}}^{2} e^{x_{E}}}{\left(e^{x_{m}} E_{m}-1\right)^{2}}\right], x=\frac{h \omega_{E}}{k_{B} T}
\end{aligned}
$$

In the above formula, $n$ is the number of atoms in the primitive cell, $k_{\mathrm{B}}$ is the Boltzmann constant, and $\theta_{\mathrm{d}}$ is the relevant Debye temperature, $m$ is an index for an optical mode of vibration. In the Debye-Einstein model the total number of modes of vibration (acoustic plus optical) is equal to the total number of atoms in the primitive unit cell. For $\mathrm{Li}_{2} \mathrm{ZnV}_{3} \mathrm{O}_{8}$ this number is 14 . In this model we have considered the ratio of the relative weights of acoustic modes and sum of the different optical modes to be $1: n-1$. Due to having two light atoms (lithium, oxygen) and two comparatively heavier atoms (vanadium and zinc) in this compound we considered two different optical modes of vibrations. The fit yields a Debye temperature of $157 \mathrm{~K}$ and Einstein tempertures of $293 \mathrm{~K}$ and $698 \mathrm{~K}$ with relative weights $C_{d}: C_{e_{1}}: C_{e_{2}}=1: 4.3: 8.7$. Upon subtracting the lattice heat capacity with the above parameters, we obtain the magnetic contribution to the heat capacity $C_{\mathrm{m}}(T)$. The entropy change $(\Delta S)$ was calculated by integrating the $C_{m} / T$ data [see Fig. 5 . The entropy change from about $25 \mathrm{~K}$ to $2 \mathrm{~K}$ is about $1.4 \mathrm{~J} / \mathrm{K}$ ( calculated for one formula unit containing $3 \mathrm{~V}^{4+}$ ions) which is only about $8 \%$ of the value for an $S=1 / 2$ system $(R \ln (2 S+1))$ which indicates the presence of many degenerate lowenergy states at low temperatures $\frac{18}{\text {. The value of the }}$ entropy change in our system is within the range of values inferred for other doped $\mathrm{LiV}_{2} \mathrm{O}_{4}$ systems ${ }^{10}$. This large reduction in the value of $\Delta S$ down to temperatures much

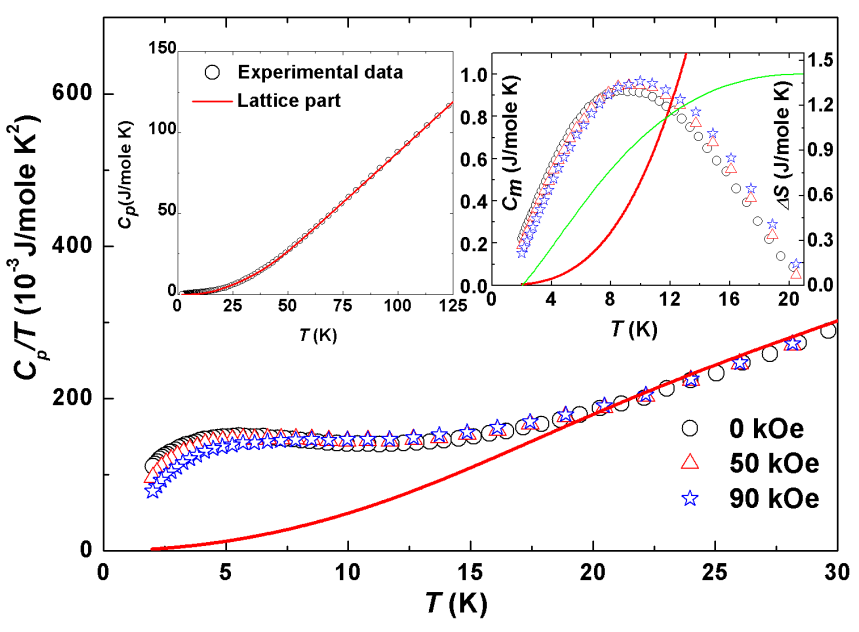

Figure 5: (Left inset) The temperature dependence of specific heat of $\mathrm{Li}_{2} \mathrm{ZnV}_{3} \mathrm{O}_{8}$; the red line represents the fitting of the heat capacity using one Debye and two Einstein terms (see text). The right inset displays the magnetic contribution of specific heat at zero field (black circle), $50 \mathrm{kOe}$ (red triangle), and $90 \mathrm{kOe}$ (blue star), the red line indicates the lattice contribution of heat capacity; the green line (right axis, left inset) shows the change of entropy calculated by integrating the $C_{\mathrm{m}} / T$ data. In the main figure $C_{P} / T$ vs. $T$ is shown to depict the slight change with the magnetic field. The red line indicates the lattice contribution in $C_{P} / T$. Note that in both the insets and in the main figure the heat capacity is calculated for one formula unit.

lower than the Weiss temperature $\left(\theta_{C W}\right)$ is typical of disordered systems and a consequence of the presence of strong geometric frustration in $\mathrm{Li}_{2} \mathrm{ZnV}_{3} \mathrm{O}_{8}$. We observed a broad maximum in the $C_{\mathrm{m}}(T)$ vs. $T$ data at $9 \mathrm{~K}$. Although we have not observed any frequency dependence in the $a c$ susceptibility data which is the main characteristic feature of canonical spin-glasses but the maximum of $C_{\mathrm{m}}(T)$ is observed above the freezing temperature $\left(T_{f}\right)$. At low $T$, (in the range of $2-5 \mathrm{~K}$ ) $C_{\mathrm{m}}(T)$ follows power law $\left(C_{\mathrm{m}}(T)=\gamma T^{\alpha}\right.$ with $\gamma=98.4 \mathrm{mJK}^{-2.2} \mathrm{~mol}^{-1}$ and $\alpha=$ 1.24) dependence with temperature. This is similar to what has been observed in other strongly correlated spinglasses 10119 . All these facts tie together to point out that there might be some formation of cluster-glass like state in the system around $9 \mathrm{~K}$ which ultimately drives the system in the metastable frozen state below $T_{f}$.

We were unable to detect the NMR signal associated with the ${ }^{51} \mathrm{~V}$ nucleus of $\mathrm{Li}_{2} \mathrm{ZnV}_{3} \mathrm{O}_{8}$. This could be due to the fact that there is a strong, on-site local moment which naturally couples well with its own nucleus. The fluctuations of this moment are very effective in causing a fast relaxation of the nuclear magnetization. This makes the detection of its NMR signal difficult. In $\mathrm{Cs}_{2} \mathrm{CuCl}_{4}{ }^{20}$ and in $\mathrm{BaV}_{3} \mathrm{O}_{8} \sqrt{21}$ as well, an NMR signals from the ${ }^{63,65} \mathrm{Cu}$ and from magnetic $\mathrm{V}^{4+}$ nuclei, respectively were not detected probably for similar reasons. On the other hand, we did not face any difficulty in observing the NMR sig- 
nal from the ${ }^{7} \mathrm{Li}$ nucleus in $\mathrm{Li}_{2} \mathrm{ZnV}_{3} \mathrm{O}_{8}$. However, we did not observe any temperature dependence of shift in the ${ }^{7} \mathrm{Li}$ spectra down to $6 \mathrm{~K}$. From $6 \mathrm{~K}$ to $4 \mathrm{~K}$ (temperature close to the freezing temperature) a small shift has been observed in the ${ }^{7} \mathrm{Li}$ spectra. We point out that in all the $\mathrm{LiV}_{2} \mathrm{O}_{4}$ based spinels reported in literature, a significant temperature dependence of the shift (scaling with the spin susceptibility) was observed. A weak hyperfine coupling may be a reason for not getting any temperature dependence of shift in $\mathrm{Li}_{2} \mathrm{ZnV}_{3} \mathrm{O}_{8}$ though that would be unusual since it has the same structure as $\mathrm{LiV}_{2} \mathrm{O}_{4}$ and one expects to have at least half the $\mathrm{Li}$ at the $\mathrm{A}$ site.

The ${ }^{7} \mathrm{Li}$ NMR linewidth increases with decreasing temperature as shown in the inset of Fig. 6. Down to about $100 \mathrm{~K}$, the full-width-at-half-maximum (FWHM) of the ${ }^{7} \mathrm{Li}$ spectrum for $\mathrm{Li}_{2} \mathrm{ZnV}_{3} \mathrm{O}_{8}$ is similar to that for pristine $\mathrm{LiV}_{2} \mathrm{O}_{4}$ and also not much different from the other doped- $\mathrm{LiV}_{2} \mathrm{O}_{4}$ systems. With a decrease in temperature the spectrum of $\mathrm{Li}_{2} \mathrm{ZnV}_{3} \mathrm{O}_{8}$ broadens at a significantly slower rate compared to $\mathrm{LiV}_{2} \mathrm{O}_{4}$ and the doped$\mathrm{LiV}_{2} \mathrm{O}_{4}$ systems 11 . We now discuss the origin of ${ }^{7} \mathrm{Li}$ NMR linewidth in $\mathrm{Li}_{2} \mathrm{ZnV}_{3} \mathrm{O}_{8}$. There are two contributions to the NMR linewidth. The first one originates from the nuclear-nuclear dipolar interaction while the second arises from the demagnetizing field due to the neighbouring powder grains 22. By using the Gaussian approximation we can write the ${ }^{7} \mathrm{Li}$ linewidth (FWHM) as

$$
\Delta=2.35 \sqrt{\langle\Delta \nu\rangle^{2}+\left(B \chi_{V} H \gamma / 2 \pi\right)^{2}}
$$

where $\langle\Delta \nu\rangle$ is the dipolar interaction term calculated from the internuclear interaction, $\chi_{V}$ is the volume susceptibility $\left(\chi_{V}=\chi_{M} d / M, d=4.134 \mathrm{~g} / \mathrm{cm}^{3}\right.$ is the density, and $M=360.09 \mathrm{~g} / \mathrm{mol}$ is the molar mass), $\gamma / 2 \pi=1654.6 \mathrm{~Hz} \mathrm{G}{ }^{-1}, H=93954.3$ Oe and $B$ is the fractional root-mean-square deviation of the local field from the applied field $H$. The temperature independent dipolar contribution has been calculated for $\mathrm{LiV}_{2} \mathrm{O}_{4}$ in Ref. ${ }^{23}$ and is sure to be smaller in $\mathrm{Li}_{2} \mathrm{ZnV}_{3} \mathrm{O}_{8}$ since it is vanadium deficient and $\mathrm{Zn}$ does not have a nuclear moment. In the first approximation, we take the dipolar width to be the same as in $\mathrm{LiV}_{2} \mathrm{O}_{4}$ and fit the FWHM of $\mathrm{Li}_{2} \mathrm{ZnV}_{3} \mathrm{O}_{8}$ to equation 1 with $B$ as a fitting parameter. For $B=9.8$ the calculated curve agrees well with the experimental data above $30 \mathrm{~K}$ (see Fig 6). At lower temperatures the discrepancy between the experimental data and the calculated curve might be due to (a) an extrinsic paramagnetic contribution to the measured susceptibility which becomes larger at lower temperatures and (b) an insufficient spectral width of the NMR pulses larger linewidth at low-temperatures leading to an underestimation of the experimental FWHM. The overall behaviour is similar to that seen in Ref. ${ }^{23}$ and hence we conclude that here as well, the ${ }^{7} \mathrm{Li}$ FWHM is dominated by macroscopic magnetisation effects.

We next report the ${ }^{7} \mathrm{Li}$ spin-lattice relaxation rate data. In $\mathrm{Li}_{2} \mathrm{ZnV}_{3} \mathrm{O}_{8}$, lithium $(I=3 / 2)$ is in the tetrahedral (A-site) and octahedral (B-site) environment with

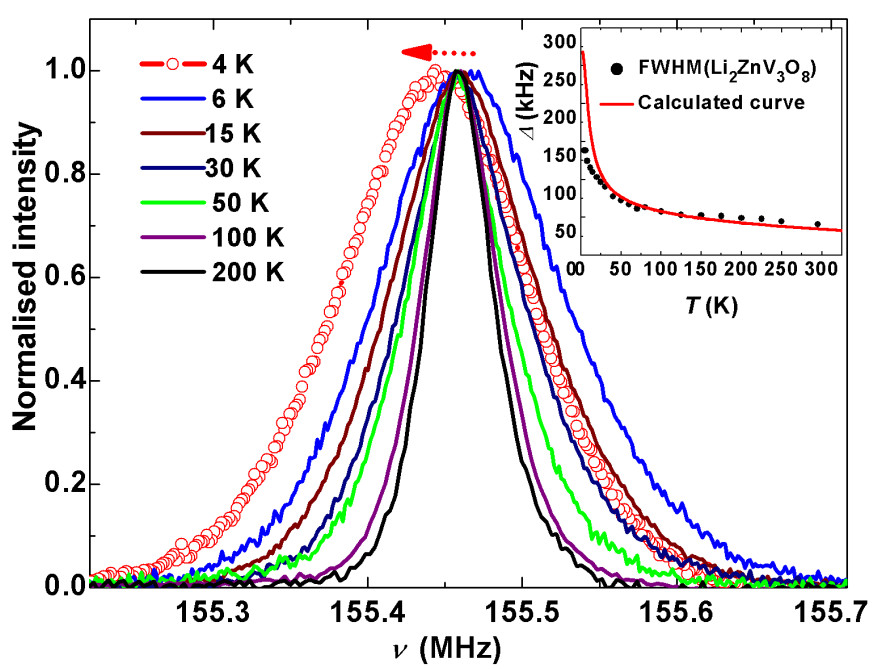

Figure 6: The ${ }^{7} \mathrm{Li}$ spectra of $\mathrm{Li}_{2} \mathrm{ZnV}_{3} \mathrm{O}_{8}$ at temperatures down to $4 \mathrm{~K}$. The dotted arrow points to the shift from $6 \mathrm{~K}$ to $4 \mathrm{~K}$. The inset shows the change in the FWHM (full width at half-maximum) with the variation in $T$. The red solid line in the inset indicates the calculated results discussed in the text.

oxygen. At least at the tetrahedral sites (but also at the octahedral sites in case of distortions) a non-zero electric field gradient (EFG) quadrupolar splitting is expected. However, down to $4 \mathrm{~K}$, we were able to irradiate the full spectrum. We have measured the recovery of the longitudinal ${ }^{7} \mathrm{Li}$ nuclear magnetization $M_{t}$ after a saturating comb and fitted it using the following stretched exponential function,

$$
1-M_{t} / M_{\infty}=A \times e^{-\left(t / T_{1}\right)^{\beta}}
$$

Here $A$ stands for the amount of saturation and $\beta$ denotes the stretching exponent. The systems which are driven into a spin-glass-like state possess a distribution of spin-lattice relaxation times due to different relaxation channels with different $T_{1}$. In these situations $\beta$ is a measure for the width of the distribution. This stretched exponential behavior of the saturation recovery of the spin-lattice relaxation data gives an indication of the presence of the local moments, although sensed very weakly through the window of the lithium nucleus. The ${ }^{7} \mathrm{Li}$ NMR $1 / T_{1}$ is nearly unchanged with temperature ${ }^{24}$ which is again surprising in light of the published data on pure and doped $\mathrm{LiV}_{2} \mathrm{O}_{4}$ (see Fig. 7 where literature data are shown along with our data). We observed an increase in our ${ }^{7} \mathrm{Li} \mathrm{NMR} 1 / T_{1}$ data for $\mathrm{Li}_{2} \mathrm{ZnV}_{3} \mathrm{O}_{8}$ near the spinglass/freezing temperature ( $4 \mathrm{~K}$ ) and a small hump-like anomaly around $50 \mathrm{~K}$ (see Fig 8). Likewise in case of $\mathrm{Li}_{1-x} \mathrm{Zn}_{x} \mathrm{~V}_{2} \mathrm{O}_{4}$ and $\mathrm{Li}\left(\mathrm{V}_{1-y} \mathrm{Ti}_{y}\right)_{2} \mathrm{O}_{4}$, an anomaly/peak in the $T$-dependence of ${ }^{7} \mathrm{Li} \mathrm{NMR} 1 / T_{1}$ was seen near the spin-glass/freezing temperature. Note that the typical value of ${ }^{7} \mathrm{Li} \mathrm{NMR} 1 / T_{1}$ data in $\mathrm{Li}_{2} \mathrm{ZnV}_{3} \mathrm{O}_{8}\left(\sim 8 s^{-1}\right)$ is vastly smaller than in any of the other vanadium based 


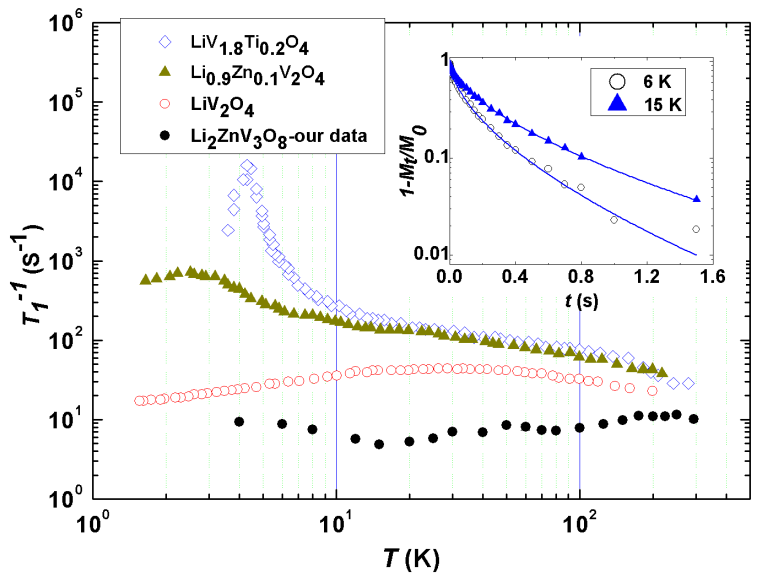

Figure 7: The spin-lattice relaxation rate $\left(1 / T_{1}\right)$ of ${ }^{7} \mathrm{Li}$ in $\mathrm{Li}_{2} \mathrm{ZnV}_{3} \mathrm{O}_{8}$ is compared to that in pure and doped $\mathrm{LiV}_{2} \mathrm{O}_{4}$ compounds from literature. In this graph $\mathrm{Li}_{2} \mathrm{ZnV}_{3} \mathrm{O}_{8}$, $\mathrm{LiV}_{2} \mathrm{O}_{4}, \mathrm{Li}_{1-x} \mathrm{Zn}_{x} \mathrm{~V}_{2} \mathrm{O}_{4}(x=0.1)$ and $\mathrm{Li}\left(\mathrm{V}_{1-y} \mathrm{Ti}_{y}\right)_{2} \mathrm{O}_{4}(y=$ $0.1)$ data are shown in black closed circle, red open circle, dark yellow closed triangle and blue open diamond symbols, respectively. In the right inset the saturation recovery data for ${ }^{7} \mathrm{Li}$ nuclear magnetisation are shown at $6 \mathrm{~K}$ and $15 \mathrm{~K}$ are shown. Also shown are the stretched exponential fits by the blue solid lines.

spinels stated above. In ${ }^{7} \mathrm{Li} \mathrm{NMR}$, the only indication of the presence of local moments in $\mathrm{Li}_{2} \mathrm{ZnV}_{3} \mathrm{O}_{8}$ is in the temperature dependence of the linewidth and in the stretched exponential behavior in the spin-lattice relaxation.

Very recently ${ }^{7} \mathrm{Li}$ NMR studies of a newly-found valence-bond geometrically-frustrated $(S=1 / 2)$ cluster magnet system $\left(\mathrm{LiZn}_{2} \mathrm{Mo}_{3} \mathrm{O}_{8}\right)$ with strong correlation $\left(\theta_{C W} \simeq-220 \mathrm{~K}\right)$ between $\mathrm{Mo}_{3} \mathrm{O}_{13}$ clusters via oxygen bridges has been reported where no shift has been observed down to $4.2 \mathrm{~K}$ and an almost temperatureindependent spin-lattice relaxation rate $\left(1 / T_{1}\right)$ of the order of $4 s^{-1}$ has been observed for the main peak ${ }^{25}$ similar to what we have seen in $\mathrm{Li}_{2} \mathrm{ZnV}_{3} \mathrm{O}_{8}$.

\section{CONCLUSION}

In this work we have reported a new vanadium-based cubic spinel $\left(\mathrm{AB}_{2} \mathrm{O}_{4}\right)$ system $\mathrm{Li}_{2} \mathrm{ZnV}_{3} \mathrm{O}_{8}$, its preparation, crystal structure, magnetic properties, specific heat properties, and NMR measurements. Among the B-site doped $\mathrm{LiV}_{2} \mathrm{O}_{4}$ systems, this is possibly the first compound where all the vanadium ions are in the $4^{+}$oxidation state. Whereas the B-sites form a frustrated lattice only $75 \%$ are occupied by $\mathrm{V}^{4+}$ and the others have nonmagnetic ions. A Curie-Weiss fit of the magnetic susceptibility data yields a Curie constant close to that expected for $S=1 / 2$. The Curie-Weiss temperature $\theta_{C W}=-214 \mathrm{~K}$ (greater than in the other $\mathrm{LiV}_{2} \mathrm{O}_{4}$ variants) is sugges-

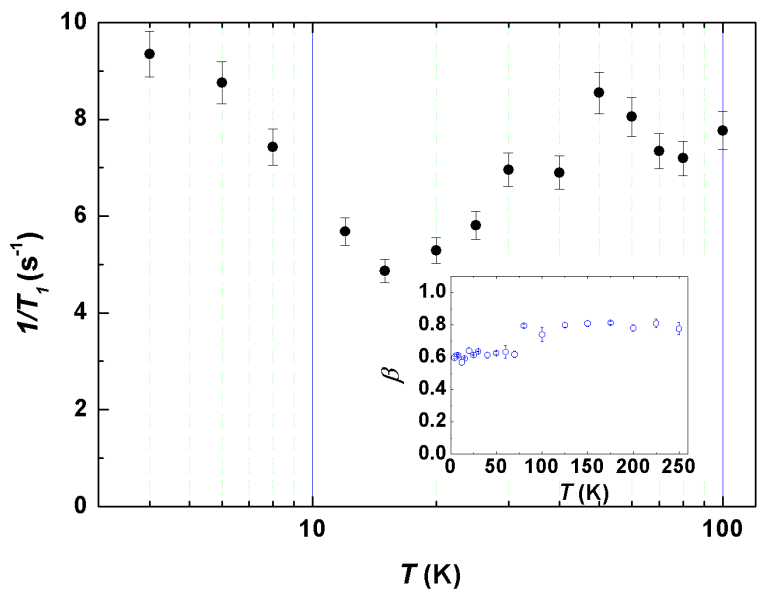

Figure 8: The temperature dependence of the ${ }^{7} \mathrm{Li}$ spin-lattice relaxation rate $\left(1 / T_{1}\right)$ is shown. The inset shows the variation of the stretching exponent $\beta$ with temperature.

tive of antiferromagnetic correlation in the system. Spin freezing is observed below $6 \mathrm{~K}$, similar to that in other $\mathrm{LiV}_{2} \mathrm{O}_{4}$ variants. The peak of the $\mathrm{ZFC}$ curve $(T \sim 3.5 \mathrm{~K})$ appears lower than the bifurcation point of the $\mathrm{ZFC} / \mathrm{FC}$ curves $(T \sim 6 \mathrm{~K})$ indicating that with decreasing temperature there might be an onset of spin-cluster formation before the system is ultimately driven into the frozen state. In the heat capacity measurement an anomaly is seen around $9 \mathrm{~K}$ and the entropy change $\Delta S$ is only $8 \%$ of that expected for an ordered $S=1 / 2$ system. This is likely due to the presence of strong geometric frustration in the system. We were unable to detect the NMR signal associated with the ${ }^{51} \mathrm{~V}$ nucleus of $\mathrm{Li}_{2} \mathrm{ZnV}_{3} \mathrm{O}_{8}$ due to strong on-site local moment so we worked with the ${ }^{7} \mathrm{Li}$ nucleus. No temperature dependence of ${ }^{7} \mathrm{Li}$ NMR shift (except near the freezing temperature of $4 \mathrm{~K}$ ) was observed which might indicate a weak hyperfine coupling with the magnetic $\mathrm{V}^{4+}$. The saturation recovery of the spin-lattice relaxation data has been fitted well using a stretched exponential function as might happen with a distribution of magnetic environments. In the temperature dependence of the spin-lattice relaxation rate we observed an increase close to the freezing temperature (4 $\mathrm{K})$ but the magnitude of the anomaly close to the freezing temperature is 2-3 orders of magnitude smaller than for $\mathrm{Li}_{1-x} \mathrm{Zn}_{x} \mathrm{~V}_{2} \mathrm{O}_{4}$ and $\mathrm{Li}\left(\mathrm{V}_{1-y} \mathrm{Ti}_{y}\right)_{2} \mathrm{O}_{4}$.

\section{ACKNOWLEDGEMENT}

Discussions with R.K.Sharma are acknowledged. The authors thank the Department of Science and Technology, Govt. of India for financial support. 
* Electronic address: tanmoyc@iitb.ac.in

† Electronic address: mahajan@phy.iitb.ac.in

1 Balents L 2010 Nature (London) 464199

2 Greedan J E 2001 J. Mater. Chem. 1137

3 Kondo S, Johnston D C, Swenson C A, Borsa F, Mahajan A V, Miller L L, Gu T, Goldman A I, Maple M B, Gajewski D A, Freeman E J, Dilley N R, Dickey R P, Merrin J, Kojima K, Luke G M, Uemura Y J, Chmaissen O and Jorgensen J D 1997 Phys. Rev. Lett. 783729

4 Chmaissem O, Jorgensen J D, Kondo S and Johnston D C 1997 Phys. rev. Lett. 794866

${ }^{5}$ Krimmel A, Loidl A, Klemm M, Horn S and Schober H 1999 Phys. Rev. Lett. 822919

6 Villain J 1979 Z. Phys. B 3331

7 Trinkl W and Loidl A, Klemm M. and Horn S 2000 Phys. Rev. B 628915

${ }^{8}$ Krimmel A, Loidl A, Klemm M, Horn S, Sheptyakov D V and Fischer P 2004 Physica B 350297

9 Miyoshi K, Ihara M, Fujiwara K and Takeuchi J 2002 Phys. Rev. B 652414

10 Brando M, B“ uttgen N, Fritsch V, Hemberger J, Kaps H, Krug von Nidda H-A , Nicklas M, Pucher K, Trinkl W, Loidl A, Scheidt E W, Klemm M and Horn S 2002 Eur. Phys. J. B 25289

11 Trinkl W, B"uttgen N, Kaps H and Loidl A 2000 Phys. Rev. B 621793

12 Okamoto Y, Nohara M, Aruga-Katori H and Tak- agi H 2007 Phys. Rev. Lett. 99137207

13 Ueda Y, Fujiwara N and Yasuoka H 1997 J. Phys. Soc. Jpn. 66778

14 Rodriguez-Carvajal J 1993 Physica B 19255

15 Pollart E 1973 Kristall and Technik 8859

16 Selwood P W 1956 Magnetochemistry (Interscience New
York)

17 Kondo S, Johnston D C and Miller L L 1999 Phys. Rev. B 592609

18 Depending on the temperature range chosen for the fit, the inferred lattice heat capacity is a bit different. Also, since this system has a large $\theta_{C W}(=-214 \mathrm{~K})$, entropy release might take place in a broad temperature range below about $200 \mathrm{~K}$. Due to the large lattice contribution at high temperatures it is nearly impossible to accurately determine the magnetic contribution to the heat capacity in this range. Consequently, there is likely to be a significant uncertainty in the entropy change estimation.

$19 \mathrm{~S}$ “ullow S, Nieuwenhuys G J, Menovsky A A, Mydosh J A, Mentink S A M, Mason T E and Buyers W J L 1997 Phys. Rev. Lett. $\mathbf{7 8} 354$

20 Vachon M A, Kundhikanjana W, Straub A, Mitrovic V F, Reyes A P, Kuhns P, Coldea R and Tylczynski Z 2006 New Journal of Physics 8222

21 Chakarabarty T, Mahajan A V, Gippius A A, Tkachev A. V, Buettgen N and Kraetschmer W 2013 Phys. Rev. B 88 014433

${ }^{22}$ Mahajan A V, Sala R, Lee E, Borsa F, Kondo S and Johnston D C 1998 Phys. Rev. B $\mathbf{5 7} 8890$

23 Onoda M , Imai H, Amako Y and Nagasawa H 1997 Phys. Rev. B 563760

24 The small change in $\beta$ seen around $80 \mathrm{~K}$ might be an artifact related to the changing of cryogen from nitrogen to helium and the need to use lower $r f$ power at lower temperatures.

25 Sheckelton J P, Foronda F R, Pan L, Moir C, McDonald R D, Lancaster T, Baker P J and Armitage N P 2014 Phys. Rev. B 89064407 